\section{Category}

Synthesis of Natural

Products and

Potential Drugs

\section{Key words}

\section{(+)-resiniferatoxin}

\section{daphnane} diterpenoid

[5+2]-oxidopyrylium cycloaddition

zirconocenemediated cyclization

\section{Achmatowicz}

reaction

orthoester formation

P. A. WENDER*, C. D. JESUDASON, H. NAKAHIRA, N. TAMURA, A. L. TEBBE, Y. UENO (STANFORD UNIVERSITY, USA)

The First Synthesis of a Daphnane Diterpene: The Enantiocontrolled Total Synthesis of (+)-Resiniferatoxin

J. Am. Chem. Soc. 1997, 119, 12976-12977.

\title{
The First Total Synthesis of (+)-Resiniferatoxin
}

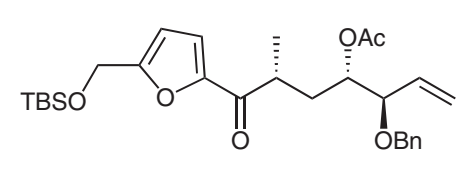

A

(7 steps from divinyl carbinol, $30 \%, 98 \%$ ee)
1. $\mathrm{NaBH}_{4}, \mathrm{MeOH}, 0^{\circ} \mathrm{C}$
2. $\mathrm{MCPBA}, \mathrm{THF}, 0^{\circ} \mathrm{C}$ to r.t.
$\frac{\text { 3. DMAP, py, } \mathrm{Ac}_{2} \mathrm{O}, 0^{\circ} \mathrm{C}}{97 \%}$

Achmatowicz reaction

1. $\mathrm{Cp}_{2} \mathrm{ZrCl}_{2}, n$-BuLi, THF-hexane (2:1) $-78{ }^{\circ} \mathrm{C}$ then $\mathrm{D}$, THF, $-78^{\circ} \mathrm{C}$ to r.t. then $\mathrm{AcOH}, 0^{\circ} \mathrm{C}$

2. NMO, TPAP (15 mol\%), $\mathrm{CH}_{2} \mathrm{Cl}_{2}, 4 \AA \mathrm{MS}$ 3. $\mathrm{CH}_{2} \mathrm{C}(\mathrm{Me}) \mathrm{MgBr}$, THF- $\mathrm{Et}_{2} \mathrm{O}(10: 1), 0{ }^{\circ} \mathrm{C}$

$$
\begin{gathered}
63 \% \\
\text { zirconocene-mediated cyclization }
\end{gathered}
$$

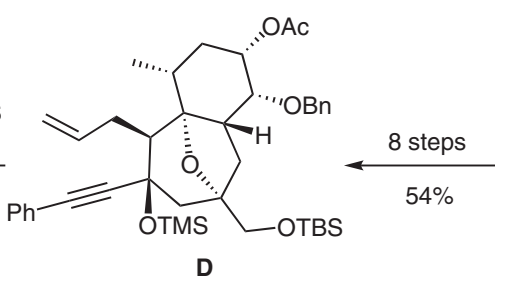

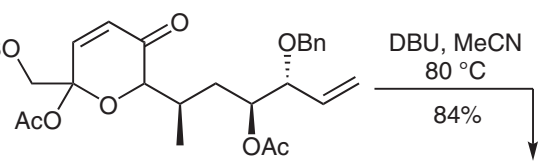

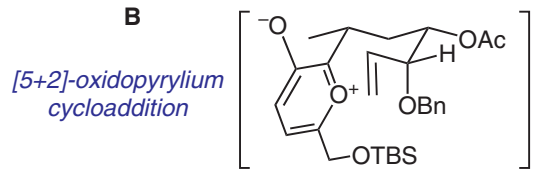

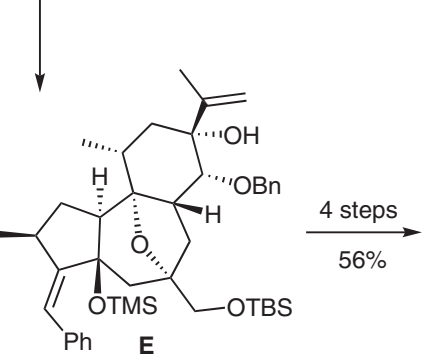

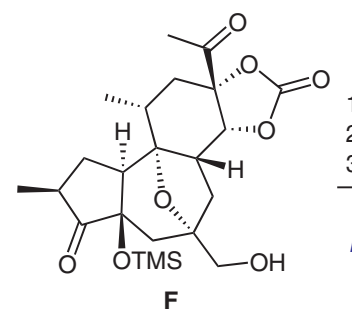

1. py, $\mathrm{Tf}_{2} \mathrm{O}, \mathrm{CH}_{2} \mathrm{Cl}_{2}, 0{ }^{\circ} \mathrm{C}$ 2. TBAI, MeCN 3. Rieke $\mathrm{Zn}, \mathrm{EtOH}, \Delta$ $88 \%$
iodoether fragmentation

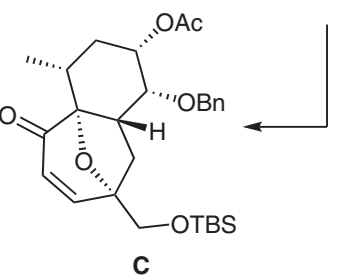

C

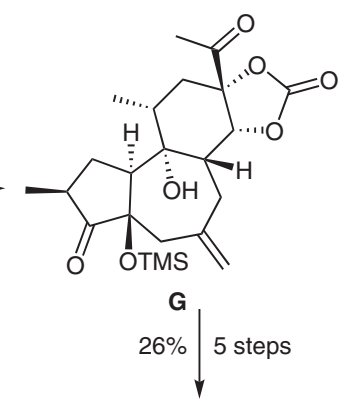

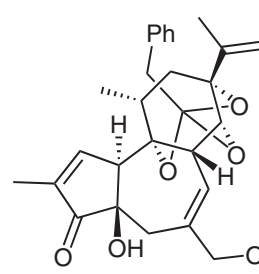

(+)-Resiniferatoxin
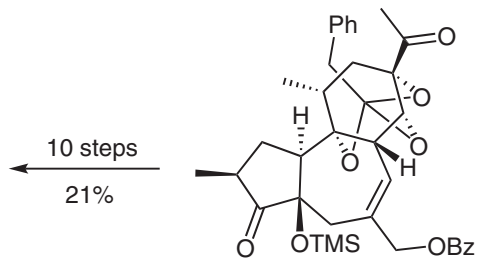

I

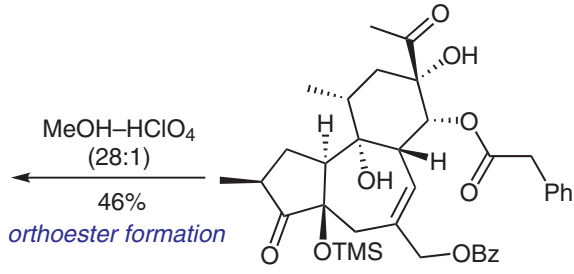

H
Significance: In 1997, Wender and co-workers reported the first total synthesis of (+)-resiniferatoxin, a daphnane diterpenoid isolated from spurge Euphorbia resinifera. Mediated by TRPV1, it exhibits analgesic potencies $10^{3}$ to $10^{5}$ greater than capsaicin. It also has a score of 16 billion on the Scoville scale.
Comment: In their seminal work, the authors employed a [5+2]-oxidopyrylium cycloaddition to generate a $[6,7]$-scaffold. $C$ was further elaborated by $\mathrm{Cp}_{2} \mathrm{ZrBu}_{2}$-mediated cyclization and reductive iodoether fragmentation en route to the target, which was completed in a total of 45 steps. 